\title{
Drosophila S2 cells as a model system to investigate mitotic spindle dynamics, architecture and function
}

\author{
Sara Moutinho-Pereira ${ }^{1, *}$, Irina Matos ${ }^{1, *}$ and Helder Maiato ${ }^{1,2}$
}

1. IBMC - Instituto de Biologia Molecular e Celular, Universidade do Porto, Rua do Campo Alegre 823, 4150-180 Porto, Portugal

2. Lab. Cell and Molecular Biology, Faculdade de Medicina, Universidade do Porto, 4200-319 Porto, Portugal

* These authors contributed equally to this work

Running Title: Spindle microtubule dynamics in Drosophila S2 cells

Keywords: Live-cell microscopy; laser microsurgery; fluorescent speckle microscopy; microtubule; microtubule drugs

\section{Correspondence:}

Helder Maiato

Instituto de Biologia Molecular e Celular,

Universidade do Porto

Rua do Campo Alegre, 823

4150-180 Porto

Portugal

Tel: $+351-22-6074900$

Fax: +351-22-6099157

E-mail: maiato@ibmc.up.pt 


\section{ABSTRACT}

In order to perpetuate their genetic content, eukaryotic cells have developed a microtubule-based machine known as the mitotic spindle. Independently of the system studied, mitotic spindles share at least one common characteristic - the dynamic nature of microtubules. This property allows the constant plasticity needed to assemble a bipolar structure, make proper kinetochoremicrotubule attachments, segregate chromosomes and finally disassemble the spindle and reform an interphase microtubule array. Here we describe a variety of experimental approaches currently used in our laboratory to study microtubule dynamics during mitosis using Drosophila melanogaster S2 cells as a model. By using quantitative live-cell imaging microscopy in combination with an advantageous labeling background, we illustrate how several cooperative pathways are used to build functional mitotic spindles. We illustrate different ways of perturbing spindle microtubule dynamics, including pharmacological inhibition and RNA interference of proteins that directly or indirectly impair microtubule dynamics. Additionally, we demonstrate the advantage of using fluorescent speckle microscopy (FSM) to investigate an intrinsic property of spindle microtubules known as poleward flux. Finally, we developed a set of laser microsurgery-based experiments that allow, with unique spatiotemporal resolution, the study of specific spindle-structures (e.g. centrosomes, microtubules and kinetochores) and their respective roles during mitosis. 


\section{INTRODUCTION}

In order to maintain their inherited genetic background, cells have developed a specialized microtubule-based structure called the mitotic spindle, which mediates the segregation of chromosomes during cell division. Many cancer therapies currently in clinical practice employ the use of drugs that target the mitotic spindle (e.g. taxanes and vinca alkaloids) with the aim of preventing cell division by blocking (and killing) cells in mitosis or leading to unviable progeny. Given its essential role, mitotic spindle assembly in animal cells is a highly conserved and redundant process that is thought to involve multiple parallel pathways (O'Connell and Khodjakov, 2007). Among these, the most wide-spread has been proposed by Kirschner and Mitchison who hypothesized that centrosomes drive spindle morphogenesis by generating astral microtubules that continuously grow and shrink, randomly "searching" for chromosomes after nuclear envelope breakdown (Kirschner and Mitchison, 1986). The increase in astral microtubule dynamics at this stage results from the phosphorylation of several regulatory microtubule-associated proteins (MAPs) as result of increased CDK1 activity (Maiato et al., 2004b; Murray, 2004). When, by chance, astral microtubules encounter a kinetochore, they are captured and become stabilized. With time, more microtubules will be gradually incorporated forming a mature kinetochore fiber (K-fiber). Despite being very attractive in its essence, this "search-andcapture" model cannot explain mitosis in cells that lack centrosomes, such as higher plants and some oocytes (Gadde and Heald, 2004; Wadsworth and Khodjakov, 2004), although some centrosome-independent "search-andcapture" events may be present in these systems (Lloyd and Chan, 2006). 
Moreover, a recent computational analysis has shown that, in a purely random fashion, the "search-and-capture" model is not sufficiently efficient to explain the capture of all kinetochores by approximately 20 microtubules in the rapid time frame of dividing animal cells (McEwen et al., 1997; Wollman et al., 2005).

Several other studies have shown that in the absence of centrosomes, animal somatic cells are able to assemble anastral spindles, either when they lack proteins that are necessary to build a functional centrosome, such as Asterless or Centrosomin (Cnn), or when this structure is physically destroyed or removed (Bonaccorsi et al., 1998; Hinchcliffe et al., 2001; Khodjakov et al., 2000a; Mahoney et al., 2006; Megraw et al., 2001; Megraw et al., 1999). In addition, a Drosophila cell line lacking centrioles could also be maintained in culture for several years (Debec et al., 1995; Debec et al., 1982), whereas mutants for cnn or Dsas-4 (a protein required for centriole duplication) are able to develop into adult flies (Basto et al., 2006; Megraw et al., 2001). One possibility could be that in these cases the self-organization of microtubules in the vicinity of chromosomes is responsible for spindle assembly, as it happens in some female meiotic systems. In this context, kinetochores would be responsible for the formation of their own K-fibers and spindle bipolarity ensured by the action of microtubule motors and cross-linking proteins (Karsenti and Vernos, 2001; Rieder, 2005). In fact, in vertebrate cells, K-fibers were observed forming in association with chromosomes without a direct connection to the centrosome or chromatin (Khodjakov et al., 2003; O'Connell et al., 2009). In agreement, studies in live Drosophila S2 cells reported that unattached kinetochores that are not facing a centrosome are able to form 
microtubules de novo, and that K-fiber growth likely occurs by microtubule addition at their kinetochore-associated end (Maiato et al., 2004a). Kinetochore-driven microtubule formation appears to rely on microtubule nucleating/stabilizing factors such as $\gamma$-tubulin, TPX2 and chromosomal passenger proteins, as well as localized RanGTP at kinetochores (Mishra et al., 2010; Torosantucci et al., 2008; Tulu et al., 2006). In conclusion, both centrosomes and kinetochores contribute to spindle assembly and acentrosomal mechanisms might always be present even in those systems normally relying on centrosomes.

More recently, it was proposed that microtubule propagation within the spindle itself could represent another mechanism to take into account in spindle morphogenesis (Mahoney et al., 2006). This idea has been reinforced after the discovery of the Augmin complex and its interaction with $\gamma$-tubulin and associated factors found in the spindle region (Goshima et al., 2008; Goshima et al., 2007; Uehara et al., 2009; Zhu et al., 2008). What remains unclear is whether this microtubule propagation pathway represents an independent molecular mechanism or whether it works together with more conventional centrosome or kinetochore-mediated pathways (Bucciarelli et al., 2009; Lawo et al., 2009).

Upon its initial formation the mitotic spindle maintains a steady-state length and shape as cells reach metaphase. However, microtubules, including those attached at kinetochores, remain dynamic and can be recycled due to turnover (Gorbsky and Borisy, 1989; Zhai et al., 1995) and poleward flux (Mitchison, 1989). This requires a labile interface that enables microtubules to slip and eventually detach from the kinetochore in response to a poleward 
force, whose origin remains controversial (Cameron et al., 2006; Dumont and Mitchison, 2009; Ganem et al., 2005; Matos et al., 2009; Miyamoto et al., 2004; Rogers et al., 2004). The importance of a labile kinetochore-microtubule interface is reflected in the capacity to correct mistakes inherent to the stochastic nature of mitotic spindle assembly and the interaction between microtubules and chromosomes (Bakhoum et al., 2009b; Ganem et al., 2005; Matos et al., 2009). In fact, increasing the stability of kinetochore-microtubule attachments in a way that errors could more difficultly be corrected is, by itself, sufficient to induce chromosomal instability in once stable diploid cells (Bakhoum et al., 2009a).

Drosophila culture cells offer a powerful set of experimental solutions to investigate and molecularly dissect mitotic spindle dynamics, architecture and function. Among these, we highlight the availability of a fully sequenced and well-annotated genome and the conservation of more than $60 \%$ of the genes with humans (Adams et al., 2000). Of particular interest for high-throughput genome-wide screenings, specific gene silencing can be easily achieved by RNA interference (RNAi) and commercial dsRNA libraries are available (Goshima et al., 2007). Additionally, Drosophila culture cells have a low chromosome number (typically between 4-12) and details of mitotic spindle morphogenesis can be easily observed at the light microscopy (LM) level by the use of available stable cell lines expressing fluorescent components of the mitotic apparatus (Mahoney et al., 2006; Maiato et al., 2004a). Finally, Drosophila culture cells are amenable for live cell microscopy and micromanipulation techniques, such as Fluorescent Speckle Microscopy (FSM) and laser microsurgery (Maiato et al., 2005; Maiato et al., 2004a; 
Matos et al., 2009). In the following sections we describe in detail some of the state-of-the-art methodologies currently in practice in our laboratory for the study and micromanipulation of microtubule organization, dynamics and function during mitosis in live Drosophila culture cells. 


\section{METHODS}

\section{Live cell microscopy analysis of mitotic spindle assembly}

The availability of several Drosophila S2 cell lines expressing various fluorescently tagged components of the mitotic apparatus allows live imaging studies in a wide-range of labeling backgrounds. This, combined with the use of RNAi (for detailed protocols see (Maiato et al., 2003; Pereira et al., 2009)) provides a powerful tool to follow different processes with a high spatiotemporal resolution in a living dividing cell. To monitor microtubule organization during spindle assembly we use a Drosophila S2 cell line stably expressing both GFP- $\alpha$-tubulin and the inner-kinetochore protein $\underline{\text { Centromere }}$ Identifier (CID) fused to mCherry. This cell line was created by transfecting S2 cells already expressing GFP- $\alpha$-tubulin (Goshima and Vale, 2003) with a pMT-CID-mCherry-BLAST vector (Coelho et al., 2008), which was derived from the original pMT-CID-GFP (Heun et al., 2006), pCo-Blast (Invitrogen) and pRSET-B-mCherry (Invitrogen) vectors. Primers used to clone mCherry and blasticidin genes with appropriate restriction sites are detailed in Table I. Alternatively, virtually any cell line expressing fluorescently tagged components of interest can be easily generated using the Gateway Cloning System (Invitrogen) after full-length cDNA amplification by PCR, using cDNA clones as template (or genomic DNA, when cDNA clones are not available) and subsequently transfected with Cellfectin reagent (Invitrogen) according to an optimized protocol (Pereira et al., 2009). For better results on stable cell line generation, it should be noted that transfection with one plasmid containing the resistance gene for selection (usually blasticidine or 
hygromycin) is significantly better than co-transfection strategies (where pCoBlast or pCoHygro are used) to avoid the problem of having cells transfected only with $\mathrm{pCoHygro/pCoBlast}$ but without the gene of interest. The latter can be cloned into a plasmid under the control of an inducible or constitutive promoter, depending on the desired expression levels for each protein. In our particular case, the copper-inducible metallothionein promoter (pMT vector [Invitrogen]) was used, but several others are available (see Invitrogen catalogue of insect expression vectors).

To investigate how Drosophila cells assemble a mitotic spindle with or without centrosomes, control and Cnn-depleted S2 cells (Mahoney et al., 2006) stably expressing GFP- $\alpha$-tubulin and CID-mCherry are plated into 0.25 $\mathrm{mg} / \mathrm{ml}$ concanavalin A-coated 22x22 $\mathrm{mm}$ coverslips and mounted in modified Rose chambers (Pereira et al., 2009) for live-cell analysis with a Spinning Disc confocal system, as described below. In control S2 cells most spindle microtubules are seen forming from the centrosomes (Fig. 1A, time 00:00). In cells with reduced Cnn after RNAi (therefore without functional centrosomes), microtubules are in close association with chromosomes and kinetochores since early prometaphase (Fig. 1B, time 00:00) and the spindle is built insideout. In both cases, fully functional mitotic apparatuses are assembled, which efficiently segregate chromosomes into two daughter cells (Fig 1A, time 20:00 and B, time 25:00). Importantly, the use of an agar overlay to flatten S2 cells (Pereira et al., 2009) may be beneficial to reveal undisclosed aspects of spindle assembly, such as acentrosomal microtubule organization around chromatin in cells containing functional centrosomes (Fig. 1C-C') or de novo microtubule formation from kinetochores (Fig. 2). 
For image acquisition, four-dimensional data sets are collected with an Andor Revolution Spinning Disc confocal system (Andor) equipped with an Electron Multiplying CCD iXonem+ camera and a Yokogawa CSU-22 unit based on an Olympus IX81 inverted microscope. Two laser lines (488 and $561 \mathrm{~nm}$ ) are used for near simultaneous excitation of GFP and mCherry and the system is driven by Andor IQ software. Typically, laser intensity range is 8-12 (arbitrary units) for GFP and 35-40 for mCherry, depending on the protein expression level. Time-lapse image stacks were collected every 30 seconds with $0.5 \mu \mathrm{m}$ z-steps and projected as maximum pixel intensities. For combination of GFP fluorescence with DIC we collect image series every 30 seconds using a Nikon Eclipse TE2000U DIC inverted wide-field microscope equipped with a CoolSnap HQ2 camera (Photometrics, Tucson, AZ). Timelapse data sets are subsequently blind deconvolved with AutoDeblur X2 software (Media Cybernetics). Additional image processing steps are performed using Image J 1.38x software (http://rsb.info.nih.gov/ij; NIH, Bethesda, MA) and Adobe Photoshop CS3 and Illustrator 8.0 (Adobe Systems, San Jose, CA).

\section{Ways of altering mitotic spindle dynamics}

Microtubules are macromolecular hollow cylinders formed by basic building blocks of $\alpha$-tubulin and $\beta$-tubulin heterodimers. Several mitotic poisons are known to disrupt/alter microtubule properties and are commonly used as chemotherapeutic agents. Additionally, the use of these substances in mitotic cells can prove useful to study several aspects of microtubule dynamics during mitosis (Jordan and Wilson, 1999). While some of these molecules 
block microtubule polymerization by binding to soluble tubulin (e.g. colchicine, colcemid, nocodazole and vinca alkaloids), thus preventing new polymer formation, others act by blocking microtubule disassembly through stabilization of GDP-bound tubulin (e.g. taxanes). When late prometaphase/metaphase Drosophila S2 cells are exposed to $200 \mu \mathrm{M}$ of colchicine (Sigma), microtubules that are initially present in the mitotic spindle can no longer assemble new polymer and start to depolymerize (Fig. 3A). Within approximately 20 minutes after drug addition, microtubules can no longer be detected, with K-fibers being the most resistant, and cells block in prometaphase.

Taxol binds directly to microtubules in cells (Manfredi et al., 1982), more specifically to $\beta$-tubulin, causing hyper-stabilization of microtubules. In Drosophila S2 cells exposed to $10 \mu \mathrm{M}$ of taxol (Sigma), microtubules become hyper-stable and two large asters form by recruiting microtubules from the spindle to the centrosome, while blocking cells in mitosis (Fig. 3B). However, in the presence of lower doses such as $10 \mathrm{nM}$, taxol's action creates more permissive conditions, allowing cells to enter anaphase (Fig. 3C) (Maresca and Salmon, 2009), despite a strong inhibition of microtubule dynamics (Fig 4B).

Entry and exit into mitosis is orchestrated by a series of biochemical "switches" that strongly impact on microtubule dynamics and consequently on mitotic spindle properties. One of the key regulators of early mitotic events during spindle assembly is CDK1. Conversely, CDK1 inactivation leads to a series of changes responsible for mitotic spindle disassembly and late mitotic events that remain less well understood. When cells previously blocked in 
metaphase (by adding MG132, a proteasome inhibitor; Sigma) are treated with roscovitine (seliciclib; Sigma), a broad CDK inhibitor, we expect to see the specific effect of roscovitine action on CDK1 (rather than on other CDKs that are not operating during mitosis) (Skoufias et al., 2007). In fact, immediately upon roscovitine addition to Drosophila $\mathrm{S} 2$ cells arrested in metaphase with $20 \mu \mathrm{M}$ of MG132, microtubules elongate and become stabilized, reminiscent to what happens during microtubule cytoskeleton remodeling during anaphase and telophase (Fig. 3D) (Moutinho-Pereira et al., 2009). However, as there is no degradation of securin, sister-chromatids are still held together as they would normally do until the end of metaphase, but the cytoplasm changes biochemically into an "anaphase-like" cytoplasm due to CDK1 inactivation.

Another way of altering MT dynamics is by direct interference with factors that control microtubule behavior, such as microtubule stabilizing and destabilizing molecules. When we use RNAi to knockdown KLP10A, a kinesin 13 protein, microtubules elongate due to the reduction of depolymerization activity at the poles (Fig. 3E) (Rogers et al., 2004). Conversely, when CLASP is perturbed, new tubulin molecules are not incorporated at kinetochore microtubules, while microtubule depolymerization still occurs at the poles (Maiato et al., 2005). This leads to spindle shortening and eventually to its collapse (Fig. 3F).

\section{Fluorescent Speckle Microscopy}

Different approaches have been used and optimized in order to quantitatively investigate the mitotic spindle property known as microtubule poleward flux. 
While 45 years ago Inoue and Forer followed the movement of areas of reduced birefringence generated with an ultraviolet microbeam (locally ablated microtubules) (Forer, 1965; Inoue, 1964), poleward flux has been for several years studied following a fiduciary mark within a fluorescent-labeled spindle. This mark, originally generated by photobleaching of rhodaminelabeled tubulin, would move towards one pole and the velocity of the displacement measured relative to a fixed point (Buster et al., 2007; Gorbsky and Borisy, 1989). However, the use of this approach poses some problems: as spindle microtubules turnover the fluorescence recovery may be too fast to allow the detection of movement by the bleached mark (Gorbsky and Borisy, 1989; Labbe et al., 2004). In order to circumvent this limitation, photoactivation tools were developed, where fluorescent marks are generated on microtubules by activation of caged-fluorescent tubulin on a dark background (Mitchison, 1989). As non-kinetochore microtubules more rapidly lose fluorescence, the remaining signal can be used as a fiduciary mark on kinetochore-microtubules (Ganem et al., 2005; Maffini et al., 2009; Mitchison, 1989; Waters et al., 1996; Zhai et al., 1995). With the development of FSM, today's strategies favor the tracking of small groups of fluorescent molecules and attempt to track single-fluorophores along spindle microtubules (Yang et al., 2007). Essentially FSM requires the existence of very low amounts of fluorescently-labeled tubulin that will be mixed with the endogenous unlabeled monomers and randomly co-assemble the microtubule polymer. The stochasticity of fluorescence incorporation will define the speckled appearance of microtubules, generating numerous intrinsic fiduciary marks that can be tracked with reference to the entire spindle structure (Waterman- 
Storer et al., 1998). Many important discoveries have been made due to the visualization of speckle dynamics, especially when assisted by computational tools for automatic speckle analysis (Danuser and Waterman-Storer, 2003; Yang et al., 2008; Yang et al., 2007).

All the above-mentioned approaches are currently used in different laboratories to study the nature of spindle microtubule flux. In our laboratory we mostly employ FSM to investigate this process in Drosophila S2 cells. Accordingly, we use a Drosophila S2 cell line stably expressing GFP-a-tubulin and mCherry-CID, both under the control of an inducible metallothionein promoter, whose leakiness produces only very low levels of fluorescenttubulin without induction (Figure 4). The mCherry-CID construct is used to guarantee the analysis of speckles specifically within K-fibers and has been described in a previous section. Cells are grown in concanavalin A-coated coverslips $(0.25 \mathrm{mg} / \mathrm{ml})$ and mounted in modified Rose chambers with Schneider's medium (Sigma-Aldrich) containing 10\% of FBS (Pereira et al., 2009). It is advisable to use conditioned media so that cells do not activate stress responses, which increases the probability of finding mitotic cells. Moreover, due to variable levels of expression, it is important to look for mitotic cells that have the lowest expression of GFP-a-tubulin detectable for the human eye.

To successfully achieve high resolution FSM it is important to be able to image a small number of fluorophores (2-10) within diffraction limited regions $(\sim 0.25 \mu \mathrm{m})$, while preventing photobleaching. This requires a sensitive imaging system, with efficient light collection and a low noise/high quantum efficiency camera. In our set up, time-lapse images are collected in a single 
plane every 5 seconds with a Nikon TE2000U inverted wide-field microscope equipped with a Coolsnap HQ2 CCD camera (Photometrics, Tucson, AZ) and Brightline fluorescence filters (Semrock, Rochester, NY) using a 100x 1.4 NA plan-Apochromatic DIC objective, and driven by NIS-elements software (Nikon, Japan). Because microtubule dynamics are highly dependent on temperature we perform all our recordings at $25{ }^{\circ} \mathrm{C}$, the physiological temperature for Drosophila, by using a temperature controlled Perspex case. In order to improve the signal-to-noise ratio and eliminate the out-of-focus blur, images are subsequently blind deconvolved with AutoDeblur X2 software (Media Cybernetics). Another possibility would be to use a similar set up coupled to a spinning-disk confocal and appropriate laser lines as described before (see also (Reis et al., 2009)).

Flux velocities are measured by kymograph analysis, in which a thin rectangular region along the axis of speckle movement within a K-fiber is extracted from each image in the time-lapse series and aligned sequentially to make a montage of the region over time (Fig. 4A, B). In these 2D kymographs, oblique lines corresponding to the movement of bright microtubule speckles are drawn over time (Fig. 4A', B'). The slopes of those lines reveal the velocity of speckle movement (Waterman-Storer et al., 1998). It should be noted that before kymograph analysis spindles are aligned using a guided-kymography tool written in Matlab (TheMathWorks, Inc.) to minimize the effects of cellular and spindle translation (Pereira and Maiato, 2010). 


\section{Laser microsurgery}

In recent years, laser microsurgery has been established as one of the most important tools to investigate mitosis. The mitotic apparatus is an appealing context for the use of such spatial domain techniques because it contains discrete structures that can be manipulated to address their respective roles in chromosome movement and signaling during mitosis (Fig. 5). Indeed, laser microsurgery has been seminal to elucidate the mechanistic basis of the spindle-assembly checkpoint (Rieder et al., 1995), the role of the centrosome in spindle assembly (Khodjakov et al., 2000b) and the role of KTs in chromosome movement (Khodjakov et al., 1996; Khodjakov and Rieder, 1996; McNeill and Berns, 1981). Drosophila culture cells offer the additional possibility of combining powerful live cell imaging and laser microsurgery with molecular tools such as RNAi. This has been particularly successful in the study of the molecular mechanism regulating kinetochore microtubule dynamics (Maiato et al., 2005), where laser microsurgery of K-fibers in S2 cells stably expressing GFP- $\alpha$-tubulin generates a reproducible essay characterized by K-fiber growth from their kinetochore-associated end at near flux rates (Fig. 5A) (Maiato et al., 2004a; Matos et al., 2009). Another useful application is the laser-mediated ablation of centrosomes, which allows one to investigate the molecular basis of acentrosomal spindle formation in animal somatic cells, as well as to dissect how acentrosomal spindles are maintained by ablating centrosomes after spindle assembly (Fig. 5B). For this purpose we use an S2 cell line stably expressing $\gamma$-tubulin fused with dsRed or GFP at its C-terminus. The latter can be combined with stable expression of mCherry- $\alpha-$ tubulin, which allows the simultaneous monitoring of microtubules and 
centrosomes (Moutinho-Pereira et al., 2009). Finally, the use of a kinetochore marker such as CID fused with GFP or mCherry allows the investigation of the roles played by kinetochores in chromosome motion, positioning and signaling throughout mitosis, as well as to induce the premature separation of sisterchromatids to investigate mechanisms of force production (Fig. 5C). A detailed description of our laser microsurgery set up and normal operation routines can be found elsewhere (Pereira et al., 2009). 


\section{ACKNOWLEDGEMENTS}

We thank Gohta Goshima and Monica Bettencourt-Dias for the kind gifts of cell lines and constructs used in this paper. Sara Moutinho-Pereira and Irina Matos are respectively supported by post-doctoral SFRH/BPD/63194/2009 and doctoral SFRD/BD/22020/2005 fellowships from Fundação para a Ciência e a Tecnologia (FCT) of Portugal. Work in the laboratory of Helder Maiato is supported by grants PTDC/BIA-BCM/66106/2006, PTDC/SAUOBD/66113/2006 and PTDC/SAU-GMG/099704/2008 from FCT, and the Gulbenkian Programme in the Frontiers of Life Sciences. 


\section{FIGURE LEGENDS:}

Figure 1. Live-cell imaging of spindle formation in control and Cnn depleted S2 cells. (A-B) Time-lapse sequence showing mitotic spindle assembly in S2 cells stably expressing cid-mCherry (green) and GFP-a-tubulin (red). Note that GFP channel is here shown in red to allow an easier visualization of cid. (A) Control S2 cells assemble a mitotic spindle in a "dominant" centrosomal fashion, whereas Cnn depleted cells (B) build a spindle through a purely chromatin/KT-mediated pathway, with a normal timing. (C-C') Control S2 cell stably expressing GFP- $\alpha$-tubulin under an agar overlay, which in this case leads to the physical exclusion of centrosomes (arrowheads) from a cellular region assembling a spindle in the vicinity of chromatin visualized with DIC. Note that over time both centrosome and acentrosomal spindles merge into a unified structure. Time is in min:sec; scale bar, $5 \mu \mathrm{m}$.

Figure 2. Live-cell imaging of de novo microtubule formation from kinetochores. (A) S2 cell stably expressing GFP- $\alpha$-tubulin illustrating the kinetics of formation of K-fibers from naked kinetochores without the contribution of centrosomes. (A') Higher magnification of the selected chromosome in A. Chromosomes can be inferred as fluorescent exclusion bodies. Time is in min:sec; scale bar, $5 \mu \mathrm{m}$.

Figure 3. Ways of altering microtubule dynamics in S2 cells. (A-F) Time-lapse sequence showing the effect of spindle poisons in S2 cells stably expressing mCherry-a-tubulin (black) (A-D), or specific RNAi treatments that alter spindle 
dynamics in S2 cells stably expressing GFP-a-tubulin (E-F). (A) Metaphase spindle of S2 cells before and after addition of $200 \mu \mathrm{M}$ of colchicine. S2 cells treated with $10 \mu \mathrm{M}$ taxol (B) and $10 \mathrm{nM}$ taxol (C) show different outcomes. Note that $\mathrm{nM}$ doses of taxol allow cells to enter anaphase. (D) Cell arrested with $20 \mu \mathrm{M}$ of MG132 for $2 \mathrm{~h}$ before and after addition $50 \mu \mathrm{M}$ of roscovitine. Microtubule dynamics is easily altered after KLP10A (E) or CLASP (F) RNAi. Note the elongated microtubules in the former treatment and their shortening in the latter. Time is in min:sec; time zero is the moment of addition of the drug (A, B and D), anaphase onset ( $C$ and $E)$ or nuclear envelope breakdown (F); scale bars, $5 \mu \mathrm{m}$.

Figure 4. FSM of kinetochore microtubules in S2 cells. S2 cells stably expressing low levels of GFP-a-tubulin and mCherry-CID are used to study poleward flux. The images in A and B correspond to time-lapse series of Control (A) and 10nM taxol (B) treated cells (reprinted from Matos et al., 2009). The white box delimits the kinetochore microtubules chosen to mount the kymographs and follow speckles through time. Note the slopes obtained when flux is present in control cells $\left(A^{\prime}\right)$ and after down regulation by taxol treatment (B'). Horizontal bar $5 \mu \mathrm{m}$; vertical bar, 1 min.

Figure 5. Laser microsurgery in S2 cells stably expressing fluorescent components of the mitotic apparatus. (A) Laser-mediated ablation of an individual K-fiber in an S2 cell stably expressing GFP- $\alpha$-tubulin. Note the regrowth of the chromosome-associated fragment, whose newly generated minus-ends remained stable after surgery. Time is in min:sec. (B) Laser- 
mediated ablation of centrosomes after spindle formation in an S2 cell stably expressing $\gamma$-tubulin-dsRed. (C) Laser-mediated separation of sisterchromatids. Note their subsequent poleward migration as indicated by the kinetochore marker CID-GFP (arrowheads) stably expressed in S2 cells. Time is in min:sec. Scale bars, $5 \mu \mathrm{m}$.

Table I. List of oligonucleotides used and respective sequences.

\begin{tabular}{l|l} 
Primer & Sequence $\left(\mathbf{5}^{\prime} \rightarrow \mathbf{3}^{\prime}\right)$ \\
\hline mCherry-Xhol F & CCGCTCGAGCGGTATGGTGAGCAAGGGCGAGGAGG \\
mCherry-Sacll R & TCCCCGCGGGGATTACTTGTACAGCTCGTCCATGC \\
Blast-Sall F & ACGCGTCGACGTCTGTTGGAATATACTATTCAACC \\
Blast-Sall R & ACGCGTCGACGTCCCGATCCAGACATGATAAGATA
\end{tabular}




\section{REFERENCES:}

Adams, M. D., Celniker, S. E., Holt, R. A., Evans, C. A., Gocayne, J. D., Amanatides, P. G., Scherer, S. E., Li, P. W., Hoskins, R. A., Galle, R. F., George, R. A., Lewis, S. E., Richards, S., Ashburner, M., Henderson, S. N., Sutton, G. G., Wortman, J. R., Yandell, M. D., Zhang, Q., Chen, L. X., Brandon, R. C., Rogers, Y. H., Blazej, R. G., Champe, M., Pfeiffer, B. D., Wan, K. H., Doyle, C., Baxter, E. G., Helt, G., Nelson, C. R., Gabor, G. L., Abril, J. F., Agbayani, A., An, H. J., Andrews-Pfannkoch, C., Baldwin, D., Ballew, R. M., Basu, A., Baxendale, J., Bayraktaroglu, L., Beasley, E. M., Beeson, K. Y., Benos, P. V., Berman, B. P., Bhandari, D., Bolshakov, S., Borkova, D., Botchan, M. R., Bouck, J., Brokstein, P., Brottier, P., Burtis, K. C., Busam, D. A., Butler, H., Cadieu, E., Center, A., Chandra, I., Cherry, J. M., Cawley, S., Dahlke, C., Davenport, L. B., Davies, P., de Pablos, B., Delcher, A., Deng, Z., Mays, A. D., Dew, I., Dietz, S. M., Dodson, K., Doup, L. E., Downes, M., DuganRocha, S., Dunkov, B. C., Dunn, P., Durbin, K. J., Evangelista, C. C., Ferraz, C., Ferriera, S., Fleischmann, W., Fosler, C., Gabrielian, A. E., Garg, N. S., Gelbart, W. M., Glasser, K., Glodek, A., Gong, F., Gorrell, J. H., Gu, Z., Guan, P., Harris, M., Harris, N. L., Harvey, D., Heiman, T. J., Hernandez, J. R., Houck, J., Hostin, D., Houston, K. A., Howland, T. J., Wei, M. H., Ibegwam, C., Jalali, M., Kalush, F., Karpen, G. H., Ke, Z., Kennison, J. A., Ketchum, K. A., Kimmel, B. E., Kodira, C. D., Kraft, C., Kravitz, S., Kulp, D., Lai, Z., Lasko, P., Lei, Y., Levitsky, A. A., Li, J., Li, Z., Liang, Y., Lin, X., Liu, X., Mattei, B., McIntosh, T. C., McLeod, M. P., McPherson, D., Merkulov, G., Milshina, N. V., Mobarry, C., Morris, J., Moshrefi, A., Mount, S. M., Moy, M., Murphy, B., Murphy, L., Muzny, D. M., Nelson, D. L., Nelson, D. R., Nelson, K. A., Nixon, K., Nusskern, D. R., Pacleb, J. M., Palazzolo, M., Pittman, G. S., Pan, S., Pollard, J., Puri, V., Reese, M. G., Reinert, K., Remington, K., Saunders, R. D., Scheeler, F., Shen, H., Shue, B. C., Siden-Kiamos, I., Simpson, M., Skupski, M. P., Smith, T., Spier, E., Spradling, A. C., Stapleton, M., Strong, R., Sun, E., Svirskas, R., Tector, C., Turner, R., Venter, E., Wang, A. H., Wang, X., Wang, Z. Y., Wassarman, D. A., Weinstock, G. M., Weissenbach, J., Williams, S. M., WoodageT, Worley, K. C., Wu, D., Yang, S., Yao, Q. A., Ye, J., Yeh, R. F., Zaveri, J. S., Zhan, M., Zhang, G., Zhao, Q., Zheng, L., Zheng, X. H., Zhong, F. N., Zhong, W., Zhou, X., Zhu, S., Zhu, X., Smith, H. O., Gibbs, R. A., Myers, E. W., Rubin, G. M., Venter, J. C., 2000. The genome sequence of Drosophila melanogaster. Science. 287, 2185-95.

Bakhoum, S. F., Genovese, G., Compton, D. A., 2009a. Deviant kinetochore microtubule dynamics underlie chromosomal instability. Curr Biol. 19, 193742.

Bakhoum, S. F., Thompson, S. L., Manning, A. L., Compton, D. A., 2009b. Genome stability is ensured by temporal control of kinetochore-microtubule dynamics. Nat Cell Biol. 11, 27-35.

Basto, R., Lau, J., Vinogradova, T., Gardiol, A., Woods, C. G., Khodjakov, A., Raff, J. W., 2006. Flies without centrioles. Cell. 125, 1375-1386. 
Bonaccorsi, S., Giansanti, M. G., Gatti, M., 1998. Spindle self-organization and cytokinesis during male meiosis in asterless mutants of Drosophila melanogaster. J Cell Biol. 142, 751-61.

Bucciarelli, E., Pellacani, C., Naim, V., Palena, A., Gatti, M., Somma, M. P., 2009. Drosophila Dgt6 interacts with Ndc80, Msps/XMAP215, and gamma-tubulin to promote kinetochore-driven MT formation. Curr Biol. 19, 1839-45.

Buster, D. W., Zhang, D., Sharp, D. J., 2007. Poleward tubulin flux in spindles: regulation and function in mitotic cells. Mol Biol Cell. 18, 3094-104.

Cameron, L. A., Yang, G., Cimini, D., Canman, J. C., Kisurina-Evgenieva, O., Khodjakov, A., Danuser, G., Salmon, E. D., 2006. Kinesin 5-independent poleward flux of kinetochore microtubules in PtK1 cells. J Cell Biol. 173, 173-9.

Coelho, P. A., Queiroz-Machado, J., Carmo, A. M., Moutinho-Pereira, S., Maiato, H., Sunkel, C. E., 2008. Dual role of topoisomerase II in centromere resolution and aurora B activity. PLoS Biol. 6, e207.

Danuser, G., Waterman-Storer, C. M., 2003. Quantitative fluorescent speckle microscopy: where it came from and where it is going. J Microsc. 211, 191207.

Debec, A., Détraves, C., Montmory, C., Géraud, G., Wright, M., 1995. Polar organization of gamma-tubulin in acentriolar mitotic spindles of Drosophila melanogaster cells. Journal of Cell Science. 108 ( Pt 7), 2645-2653.

Debec, A., Szollosi, A., Szollosi, D., 1982. A Drosophila melanogaster cell line lacking centriole. Biol Cell. 44, 133-138.

Dumont, S., Mitchison, T. J., 2009. Compression regulates mitotic spindle length by a mechanochemical switch at the poles. Curr Biol. 19, 1086-95.

Forer, A., 1965. Local Reduction of Spindle Fiber Birefringence in Living Nephrotoma Suturalis (Loew) Spermatocytes Induced by Ultraviolet Microbeam Irradiation. J Cell Biol. 25, SUPPL:95-117.

Gadde, S., Heald, R., 2004. Mechanisms and molecules of the mitotic spindle. Current Biology: CB. 14, R797-805-R797-805.

Ganem, N. J., Upton, K., Compton, D. A., 2005. Efficient mitosis in human cells lacking poleward microtubule flux. Curr Biol. 15, 1827-32.

Gorbsky, G. J., Borisy, G. G., 1989. Microtubules of the kinetochore fiber turn over in metaphase but not in anaphase. J Cell Biol. 109, 653-62.

Goshima, G., Mayer, M., Zhang, N., Stuurman, N., Vale, R. D., 2008. Augmin: a protein complex required for centrosome-independent microtubule generation within the spindle. The Journal of Cell Biology. 181, 421-429.

Goshima, G., Vale, R. D., 2003. The roles of microtubule-based motor proteins in mitosis: comprehensive RNAi analysis in the Drosophila S2 cell line. The Journal of Cell Biology. 162, 1003-1016.

Goshima, G., Wollman, R., Goodwin, S. S., Zhang, N., Scholey, J. M., Vale, R. D., Stuurman, N., 2007. Genes required for mitotic spindle assembly in Drosophila S2 cells. Science (New York, N.Y.). 316, 417-421.

Heun, P., Erhardt, S., Blower, M. D., Weiss, S., Skora, A. D., Karpen, G. H., 2006. Mislocalization of the Drosophila centromere-specific histone CID promotes formation of functional ectopic kinetochores. Dev Cell. 10, 303-15.

Hinchcliffe, E. H., Miller, F. J., Cham, M., Khodjakov, A., Sluder, G., 2001. Requirement of a centrosomal activity for cell cycle progression through G1 into S phase. Science (New York, N.Y.). 291, 1547-1550. 
Inoue, S., Organization and function of the mitotic spindle. In: R. D. Allen, N. Kamiya, Eds.), Primitive motile systems in cell biology. Academic Press, New York, 1964, pp. 549-598.

Jordan, M. A., Wilson, L., 1999. The use and action of drugs in analyzing mitosis. Methods Cell Biol. 61, 267-95.

Karsenti, E., Vernos, I., 2001. The mitotic spindle: a self-made machine. Science. 294, 543-7.

Khodjakov, A., Cole, R. W., Bajer, A. S., Rieder, C. L., 1996. The force for poleward chromosome motion in Haemanthus cells acts along the length of the chromosome during metaphase but only at the kinetochore during anaphase. $\mathrm{J}$ Cell Biol. 132, 1093-104.

Khodjakov, A., Cole, R. W., Oakley, B. R., Rieder, C. L., 2000a. Centrosomeindependent mitotic spindle formation in vertebrates. Current Biology: CB. $10,59-67$.

Khodjakov, A., Cole, R. W., Oakley, B. R., Rieder, C. L., 2000b. Centrosomeindependent mitotic spindle formation in vertebrates. Curr Biol. 10, 59-67.

Khodjakov, A., Copenagle, L., Gordon, M. B., Compton, D. A., Kapoor, T. M., 2003. Minus-end capture of preformed kinetochore fibers contributes to spindle morphogenesis. J Cell Biol. 160, 671-83.

Khodjakov, A., Rieder, C. L., 1996. Kinetochores moving away from their associated pole do not exert a significant pushing force on the chromosome. J Cell Biol. $135,315-27$.

Kirschner, M., Mitchison, T., 1986. Beyond self-assembly: from microtubules to morphogenesis. Cell. 45, 329-42.

Labbe, J. C., McCarthy, E. K., Goldstein, B., 2004. The forces that position a mitotic spindle asymmetrically are tethered until after the time of spindle assembly. J Cell Biol. 167, 245-56.

Lawo, S., Bashkurov, M., Mullin, M., Ferreria, M. G., Kittler, R., Habermann, B., Tagliaferro, A., Poser, I., Hutchins, J. R., Hegemann, B., Pinchev, D., Buchholz, F., Peters, J. M., Hyman, A. A., Gingras, A. C., Pelletier, L., 2009. HAUS, the 8-subunit human Augmin complex, regulates centrosome and spindle integrity. Curr Biol. 19, 816-26.

Lloyd, C., Chan, J., 2006. Not so divided: the common basis of plant and animal cell division. Nat Rev Mol Cell Biol. 7, 147-52.

Maffini, S., Maia, A. R., Manning, A. L., Maliga, Z., Pereira, A. L., Junqueira, M., Shevchenko, A., Hyman, A., Yates, J. R., 3rd, Galjart, N., Compton, D. A., Maiato, H., 2009. Motor-independent targeting of CLASPs to kinetochores by CENP-E promotes microtubule turnover and poleward flux. Curr Biol. 19, 1566-72.

Mahoney, N. M., Goshima, G., Douglass, A. D., Vale, R. D., 2006. Making microtubules and mitotic spindles in cells without functional centrosomes. Current Biology: CB. 16, 564-569.

Maiato, H., Khodjakov, A., Rieder, C. L., 2005. Drosophila CLASP is required for the incorporation of microtubule subunits into fluxing kinetochore fibres. Nat Cell Biol. 7, 42-7.

Maiato, H., Rieder, C. L., Khodjakov, A., 2004a. Kinetochore-driven formation of kinetochore fibers contributes to spindle assembly during animal mitosis. The Journal of Cell Biology. 167, 831-840.

Maiato, H., Sampaio, P., Sunkel, C. E., 2004b. Microtubule-associated proteins and their essential roles during mitosis. Int Rev Cytol. 241, 53-153. 
Maiato, H., Sunkel, C. E., Earnshaw, W. C., 2003. Dissecting mitosis by RNAi in Drosophila tissue culture cells. Biol Proced Online. 5, 153-161.

Manfredi, J. J., Parness, J., Horwitz, S. B., 1982. Taxol binds to cellular microtubules. J Cell Biol. 94, 688-96.

Maresca, T. J., Salmon, E. D., 2009. Intrakinetochore stretch is associated with changes in kinetochore phosphorylation and spindle assembly checkpoint activity. J Cell Biol. 184, 373-81.

Matos, I., Pereira, A. J., Lince-Faria, M., Cameron, L. A., Salmon, E. D., Maiato, H., 2009. Synchronizing chromosome segregation by flux-dependent force equalization at kinetochores. J Cell Biol. 186, 11-26.

McEwen, B. F., Heagle, A. B., Cassels, G. O., Buttle, K. F., Rieder, C. L., 1997. Kinetochore fiber maturation in PtK1 cells and its implications for the mechanisms of chromosome congression and anaphase onset. The Journal of Cell Biology. 137, 1567-1580.

McNeill, P. A., Berns, M. W., 1981. Chromosome behavior after laser microirradiation of a single kinetochore in mitotic PtK2 cells. J Cell Biol. 88, 543-53.

Megraw, T. L., Kao, L. R., Kaufman, T. C., 2001. Zygotic development without functional mitotic centrosomes. Current Biology: CB. 11, 116-120.

Megraw, T. L., Li, K., Kao, L. R., Kaufman, T. C., 1999. The centrosomin protein is required for centrosome assembly and function during cleavage in Drosophila. Development (Cambridge, England). 126, 2829-2839.

Mishra, R. K., Chakraborty, P., Arnaoutov, A., Fontoura, B. M., Dasso, M., 2010. The Nup107-160 complex and gamma-TuRC regulate microtubule polymerization at kinetochores. Nat Cell Biol.

Mitchison, T. J., 1989. Polewards microtubule flux in the mitotic spindle: evidence from photoactivation of fluorescence. J Cell Biol. 109, 637-52.

Miyamoto, D. T., Perlman, Z. E., Burbank, K. S., Groen, A. C., Mitchison, T. J., 2004. The kinesin Eg5 drives poleward microtubule flux in Xenopus laevis egg extract spindles. J Cell Biol. 167, 813-8.

Moutinho-Pereira, S., Debec, A., Maiato, H., 2009. Microtubule cytoskeleton remodeling by acentriolar microtubule-organizing centers at the entry and exit from mitosis in Drosophila somatic cells. Mol Biol Cell. 20, 2796-808.

Murray, A. W., 2004. Recycling the cell cycle: cyclins revisited. Cell. 116, 221-34.

O'Connell, C. B., Khodjakov, A. L., 2007. Cooperative mechanisms of mitotic spindle formation. J Cell Sci. 120, 1717-22.

O'Connell, C. B., Loncarek, J., Kalab, P., Khodjakov, A., 2009. Relative contributions of chromatin and kinetochores to mitotic spindle assembly. J Cell Biol. 187, 43-51.

Pereira, A. J., Maiato, H., 2010. Improved kymography tools and its applications to mitosis. Methods.

Pereira, A. J., Matos, I., Lince-Faria, M., Maiato, H., 2009. Dissecting mitosis with laser microsurgery and RNAi in Drosophila cells. Methods Mol Biol. 545, 145-64.

Reis, R., Feijao, T., Gouveia, S., Pereira, A. J., Matos, I., Sampaio, P., Maiato, H., Sunkel, C. E., 2009. Dynein and mast/orbit/CLASP have antagonistic roles in regulating kinetochore-microtubule plus-end dynamics. J Cell Sci. 122, 254353.

Rieder, C. L., 2005. Kinetochore fiber formation in animal somatic cells: dueling mechanisms come to a draw. Chromosoma. 114, 310-318. 
Rieder, C. L., Cole, R. W., Khodjakov, A., Sluder, G., 1995. The checkpoint delaying anaphase in response to chromosome monoorientation is mediated by an inhibitory signal produced by unattached kinetochores. J Cell Biol. 130, 9418.

Rogers, G. C., Rogers, S. L., Schwimmer, T. A., Ems-McClung, S. C., Walczak, C. E., Vale, R. D., Scholey, J. M., Sharp, D. J., 2004. Two mitotic kinesins cooperate to drive sister chromatid separation during anaphase. Nature. 427, 364-70.

Skoufias, D. A., Indorato, R.-L., Lacroix, F., Panopoulos, A., Margolis, R. L., 2007. Mitosis persists in the absence of Cdk1 activity when proteolysis or protein phosphatase activity is suppressed. The Journal of Cell Biology. 179, 671-685.

Torosantucci, L., De Luca, M., Guarguaglini, G., Lavia, P., Degrassi, F., 2008. Localized RanGTP accumulation promotes microtubule nucleation at kinetochores in somatic mammalian cells. Mol Biol Cell. 19, 1873-82.

Tulu, U. S., Fagerstrom, C., Ferenz, N. P., Wadsworth, P., 2006. Molecular requirements for kinetochore-associated microtubule formation in mammalian cells. Curr Biol. 16, 536-41.

Uehara, R., Nozawa, R.-s., Tomioka, A., Petry, S., Vale, R. D., Obuse, C., Goshima, G., 2009. The augmin complex plays a critical role in spindle microtubule generation for mitotic progression and cytokinesis in human cells. Proceedings of the National Academy of Sciences of the United States of America. 106, 6998-7003.

Wadsworth, P., Khodjakov, A., 2004. E pluribus unum: towards a universal mechanism for spindle assembly. Trends in Cell Biology. 14, 413-419.

Waterman-Storer, C. M., Desai, A., Bulinski, J. C., Salmon, E. D., 1998. Fluorescent speckle microscopy, a method to visualize the dynamics of protein assemblies in living cells. Curr Biol. 8, 1227-30.

Waters, J. C., Mitchison, T. J., Rieder, C. L., Salmon, E. D., 1996. The kinetochore microtubule minus-end disassembly associated with poleward flux produces a force that can do work. Mol Biol Cell. 7, 1547-58.

Wollman, R., Cytrynbaum, E. N., Jones, J. T., Meyer, T., Scholey, J. M., Mogilner, A., 2005. Efficient chromosome capture requires a bias in the 'search-andcapture' process during mitotic-spindle assembly. Current Biology: CB. 15, 828-832.

Yang, G., Cameron, L. A., Maddox, P. S., Salmon, E. D., Danuser, G., 2008. Regional variation of microtubule flux reveals microtubule organization in the metaphase meiotic spindle. J Cell Biol. 182, 631-9.

Yang, G., Houghtaling, B. R., Gaetz, J., Liu, J. Z., Danuser, G., Kapoor, T. M., 2007. Architectural dynamics of the meiotic spindle revealed by single-fluorophore imaging. Nat Cell Biol. 9, 1233-42.

Zhai, Y., Kronebusch, P. J., Borisy, G. G., 1995. Kinetochore microtubule dynamics and the metaphase-anaphase transition. J Cell Biol. 131, 721-34.

Zhu, H., Coppinger, J. A., Jang, C. Y., Yates, J. R., 3rd, Fang, G., 2008. FAM29A promotes microtubule amplification via recruitment of the NEDD1-gammatubulin complex to the mitotic spindle. J Cell Biol. 183, 835-48. 


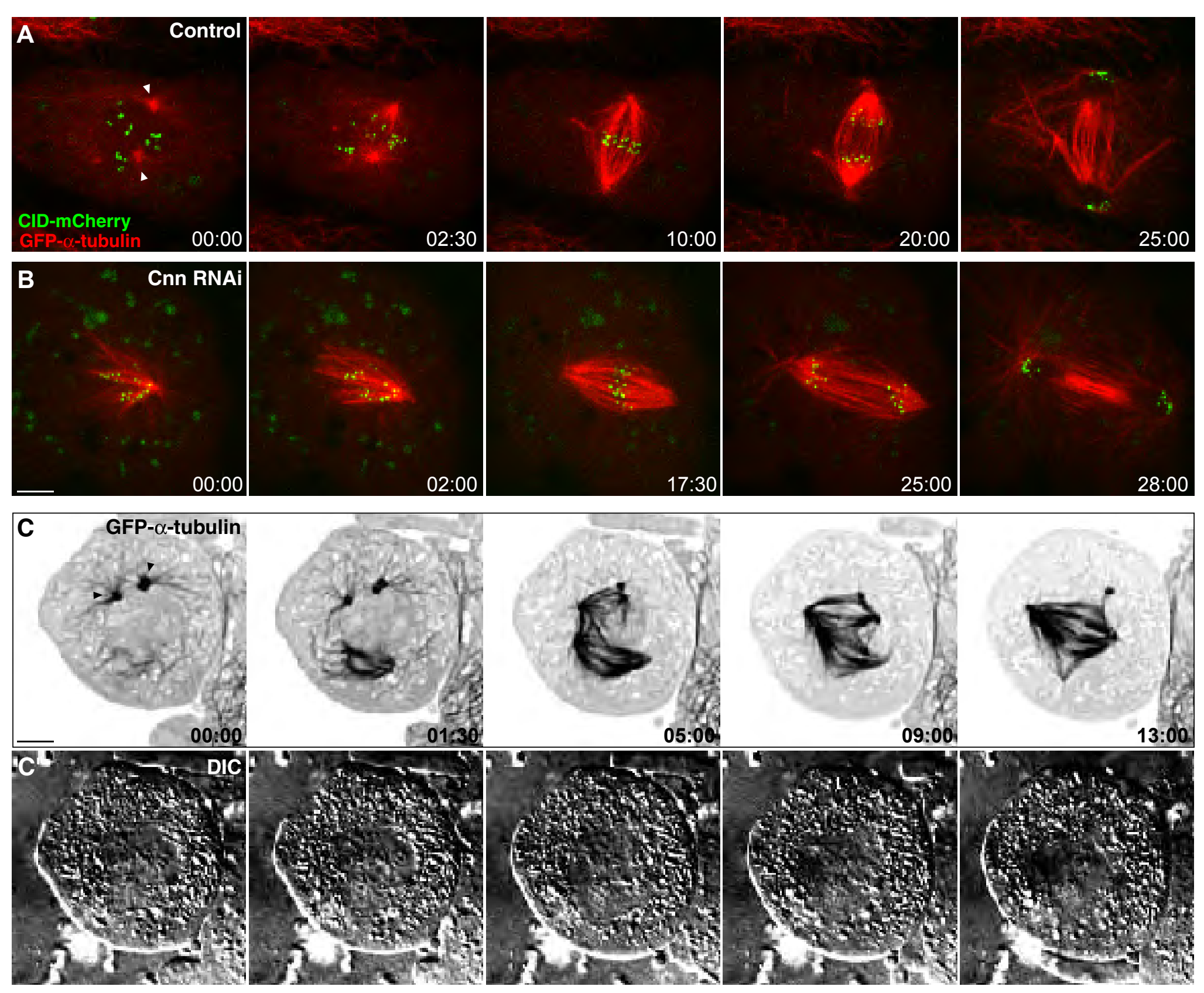




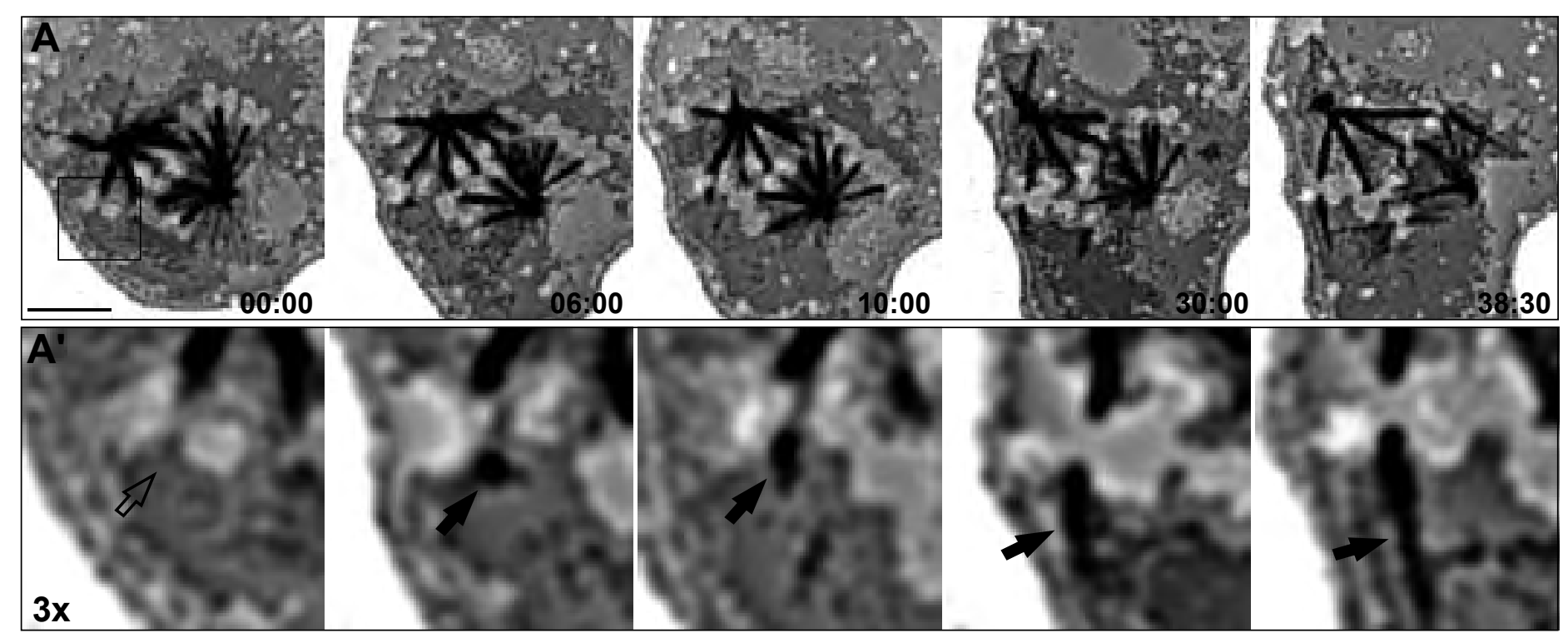




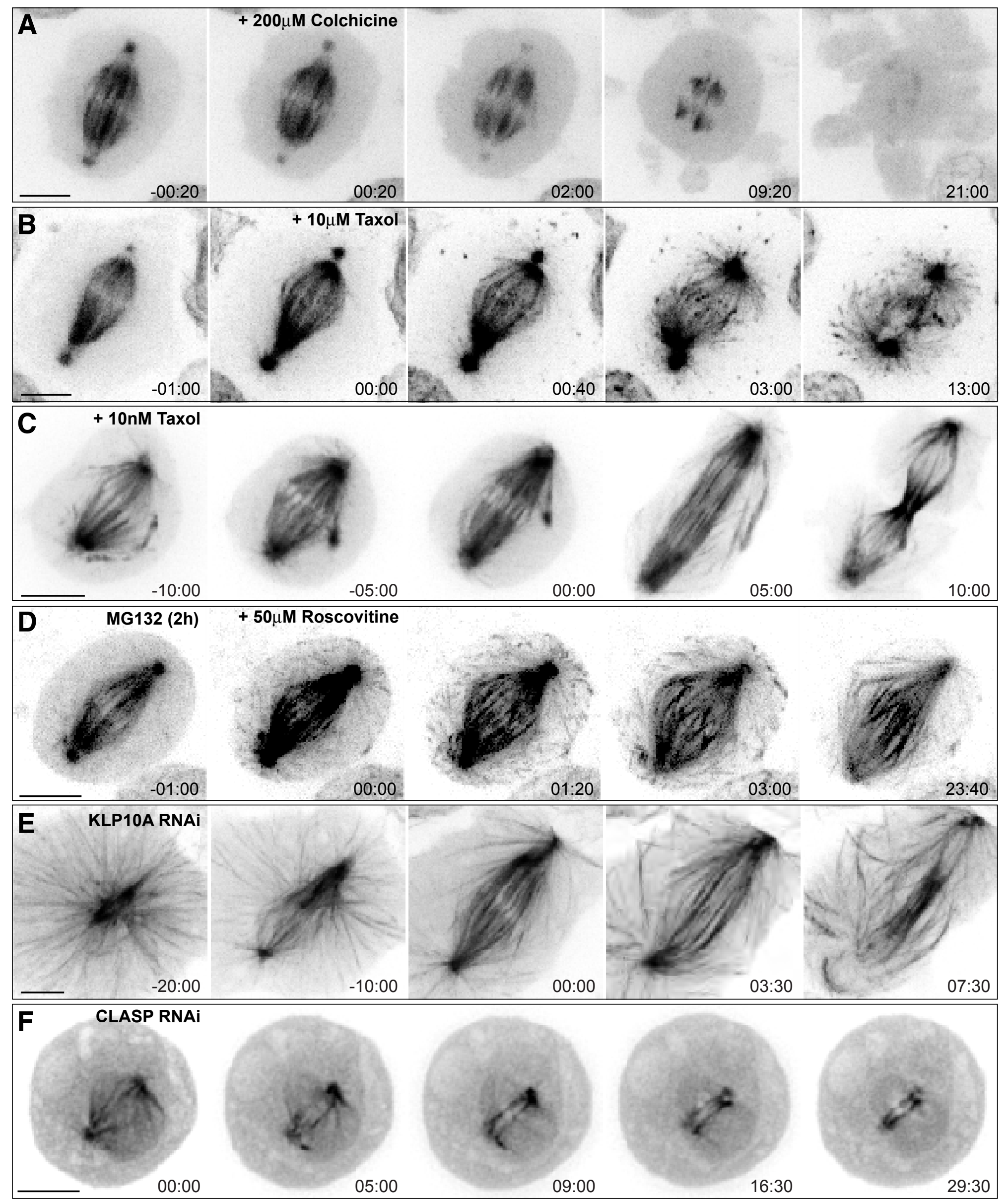



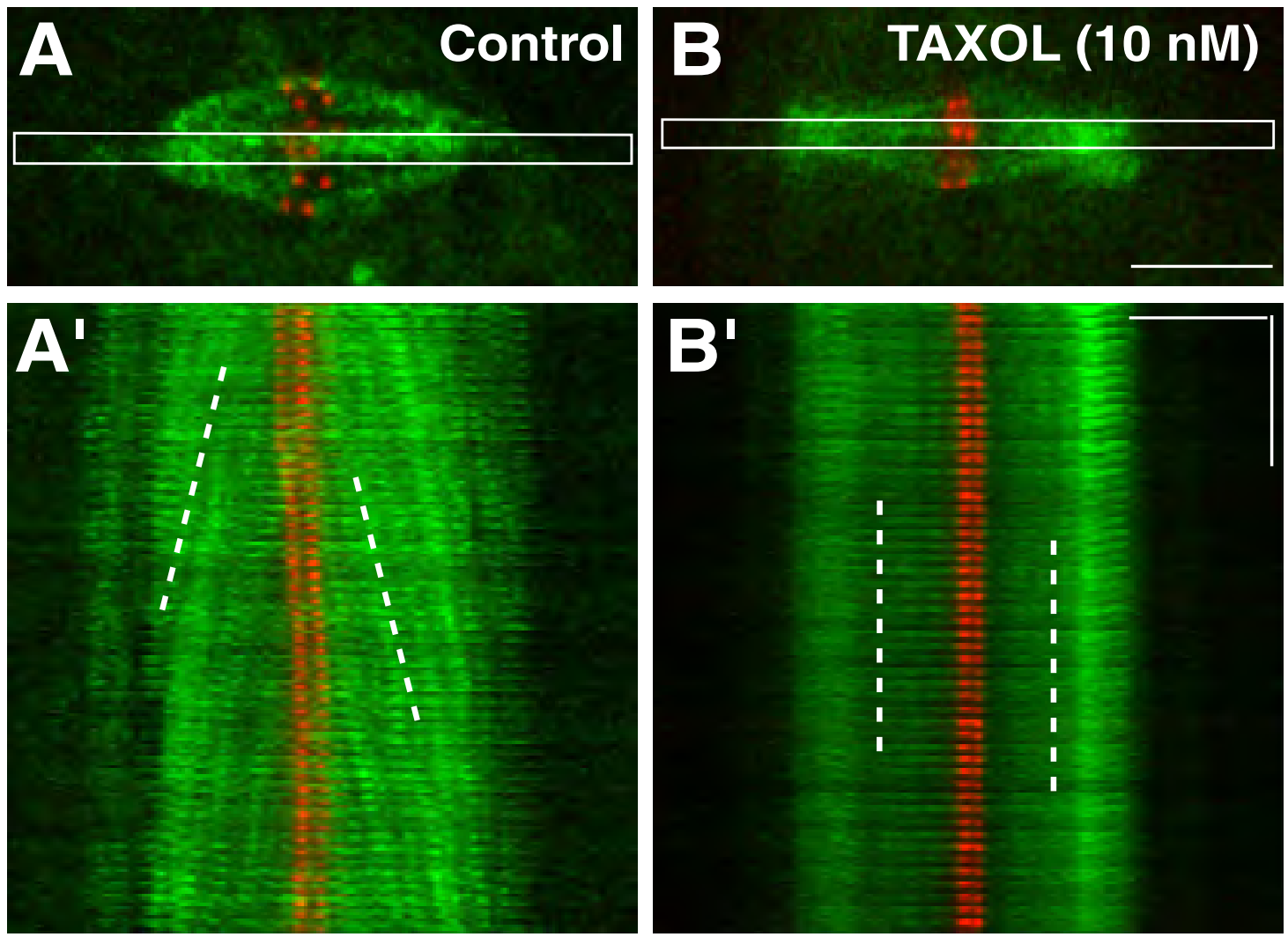


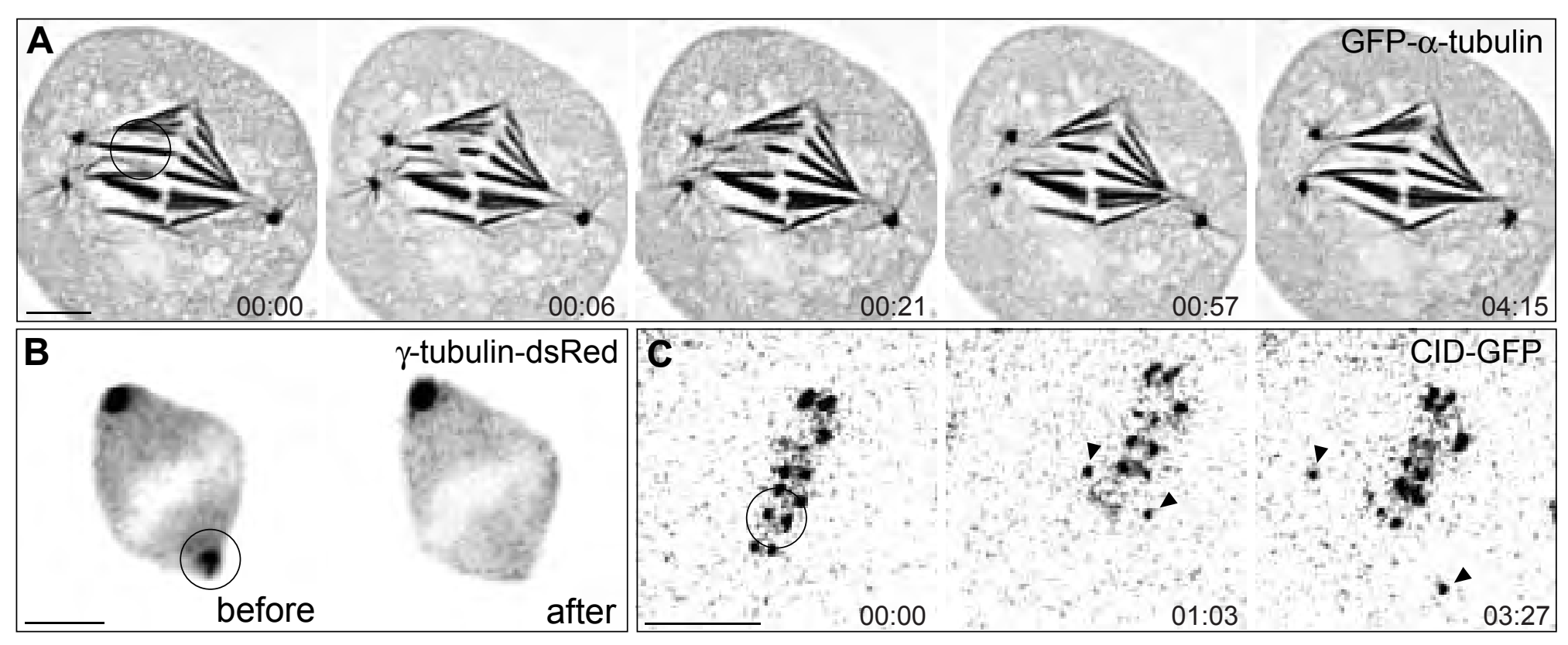

